\title{
SINERGIA
}

REVISTA DO INSTITUTO DE CIÊNCIAS ECONÔMICAS, ADMINISTRATIVAS E CONTÁBEIS (ICEAC)

\section{A GESTÃO DO CONHECIMENTO HOLÍSTICA: ANÁLISE DE ADERÊNCIA DO MODELO DE TERRA (2005)}

\author{
FÁBIO CORREA \\ FABRÍCIO ZIVIANI" \\ RENATA DE SOUZA FRANCA* \\ JUREMA SUELY DE ARAUJO NERY RIBEIRO
}

\begin{abstract}
RESUMO
Há uma diversidade de modelos de Gestão do Conhecimento apresentados na literatura. Entretanto, há uma contundente sinalização para que tais modelos sejam orientados ao paradigma holístico. Mediante esta necessidade, esta pesquisa caracteriza a Gestão do Conhecimento holística e as partes que a compõem. Esse feito tem por intento alicerçar o objetivo geral de analisar a aderência do modelo de Terra (2005) a GC holística. Para promover essa análise, a abordagem qualitativa-quantitativa foi adotada como procedimento metodológico. Por resultado, o modelo de Terra (2005) se adere em $69,2 \%$ pontos percentuais a Gestão do Conhecimento holística, conforme caracterização apresentada nesta pesquisa, permitindo concluir que há oportunidade de desenvolvimento de modelos mais abrangentes. Acredita-se que essa pesquisa tende a contribuir por delinear o que consiste a Gestão do Conhecimento holística e suas partes, criando um alicerce para o desenvolvimento de novos modelos pautados no paradigma holístico. Ademais, contribui por apresentar um método que, se aplicado a outros modelos, tende a revelar o status dessa gestão organizacional e direcionar pesquisas para que se contemple o gerenciamento do conhecimento de forma totalitária nas organizações. Assim, a aplicação do método desta pesquisa em outros modelos é uma sugestão de pesquisa futura.
\end{abstract}

Palavras-chave: Gestão do Conhecimento. Paradigma holístico. Gestão do Conhecimento holística. Terra (2005). Análise de Conteúdo.

\section{ABSTRACT}

There are a variety of Knowledge Management models presented in the literature. However, there is a strong signal that such models are oriented to the holistic paradigm. Through this need, this research characterizes the Holistic Knowledge Management and the parts that compose it. This work is intended to underpin the general objective of analyzing the adherence of the Earth (2005) model to holistic GC. To promote this analysis, the qualitative-quantitative approach was adopted as a methodological procedure. As a result, the Terra (2005) model adheres to the Holistic Knowledge Management (69.2\%) according to the characterization presented in this research, allowing the conclusion that there is an opportunity to develop more comprehensive models. It is believed that this research tends to contribute to delineate what consists of holistic Knowledge Management and its parts, creating a foundation for the development of new models based on the holistic paradigm. In addition, it contributes to present a method that, if applied to other models, tends to reveal the status of this organizational management and to direct researches so that the management of knowledge in a totalitarian way in the organizations is contemplated. Thus, the application of the method of this research in other models is a suggestion of future research.

Keywords: Knowledge management. Holistic paradigma. Holistic Knowledge Management. Terra (2005). Content analysis.

Recebido em: 24-07-2018 Aceito em: 24-01-2019

\section{INTRODUÇÃO}

Desde que as organizações e a academia passaram a reconhecer o conhecimento enquanto um ativo organizacional, diversas abordagens orientaram a má exploração desse recurso. Aprendizagem Organizacional (SENGE, 1990; GONÇALVES et al. 2016), Visão Baseada em Recursos (WERNERFELT, 1984; LEITE; AGNOLIN; CARVALHO, 2017), Capacidades Dinâmicas (TEECE; PISANO; SHUEN, 1997; ALVES et al., 2017), para citar alguns, são áreas que tratam o conhecimento como um componente de suas

\footnotetext{
"Doutor e Mestre em Sistemas de Informação e Gestão do Conhecimento

"* Doutor em Ciência da Informação pela Universidade Federal de Minas Gerais - UFMG, Mestre em Administração Pública pela Escola de Governo - Fundação João Pinheiro, professor adjunto do Programa de Pós-Graduação em Sistemas de Informação e Gestão do Conhecimento da Universidade FUMEC e professor Adjunto da Universidade do Estado de Minas Gerais

Doutoranda em Sistemas de Informação e Gestão do Conhecimento pela Universidade FUMEC e Mestra em Sistemas de Informação e Gestão do Conhecimento pela FUMEC

Doutoranda em Sistemas de Informação e Gestão do Conhecimento - FUMEC e Mestra em Administraçãopela Faculdade de Estudos Administrativos de Minas Gerias - FEAD
} 
abordagens. Entretanto, a Gestão do Conhecimento (GC) se apresenta como uma abordagem que se volta, essencialmente, para este ativo.

Não há uma definição precisa sobre a GC (VALENTIM, 2014), pois essa pode ser estudada por diversos focos e, portanto, tende a admitir diversas faces. Há estudos que discorrem sobre a epistemologia do conhecimento (NICKOLS, 2000; GRANT, 2007; CORRÊA et al., 2017) e a relação desse com a vantagem competitiva (SOUSA, PRATA, PEREIRA, 2018) e com o desempenho organizacional (RIBEIRO, et al., 2017). Sobre esses múltiplos olhares, junto ao alicerce das áreas que se propõem a considerar este ativo, emergem os modelos de GC.

Um modelo, enquanto uma estrutura que representa os elementos da $\mathrm{GC}$ e as conexões entre esses (WEBER, 2002; HEISIG, 2009), se orienta ao gerenciamento deste ativo com um foco específico, seja para a melhora do desempenho organizacional, seja para a obtenção de vantagem competitiva ou outro objetivo. Todavia, o foco em determinado desígnio acarreta em modelos variados que contemplam diferentes elementos.

Mediante a multidiversidade de modelos existentes, Holsapple e Joshi (1999), Rubenstein-Montano et al. (2001), Heisig (2009) e Fteimi (2015), cada qual em seu tempo, analisaram um total de 270 modelos de GC e concluíram haver necessidade de modelos que sejam orientados ao paradigma holístico. Essa conclusão anuncia a necessidade de se compreender o que é a GC ajustada a esse paradigma e quais as partes que a compõem. Ademais, pelo fato de os estudos anunciarem essa necessidade - de 1999 a 2015 - e mediante o expressivo número de modelos analisados - admite-se que essa é uma orientação factível de ser considerada e desenvolvida para elevar a GC a uma abordagem que seja, de fato, totalitária.

É sobre o alicerce da GC holística, enquanto um alinhamento do gerenciamento do conhecimento com o paradigma holístico no que tange a modelos de GC, que essa pesquisa se estabelece. Especificamente, busca-se investigar a seguinte questão problema: 0 modelo de Terra (2005) apresenta aderência a um modelo de GC holístico? Desse modo, em primeira instância, esta pesquisa ajusta a GC ao paradigma holístico para que, posteriormente, se possa contemplar o objetivo geral de analisar a aderência do modelo deTerra (2005) a GC holística, buscando obter uma resposta para a interrogativa supracitada.

A relevância desta pesquisa dá-se pelo fato de esse paradigma ser apontado como uma abordagem necessária a GC, conforme estudos supracitados que analisaram 270 modelos de GC longitudinalmente. Assim, explorar o que consiste a GC holística é uma alternativa para que se possa compreender o que se almeja por essa perspectiva. Mediante essa relevância, esta pesquisa se justifica por analisar o modelo de GC de Terra (2005) de forma a identificar o quanto esse se adere a GC holística, permitindo compreender como um dos principais modelos nacionais dessa área contempla o gerenciamento do conhecimento por esse paradigma.

Acredita-se que algumas contribuições são obtidas por meio desta pesquisa. Primeiramente, ao delinear o que consiste a GC holística, esse delineamento tende a contribuir com o meio acadêmico para que se orientem ao desenvolvimento de modelos que contemplem essa gestão em sua totalidade no âmbito empresarial. Em segundo momento, ao analisar o modelo de Terra (2005), esta pesquisa contribui por apresentar um método de análise que, se aplicado a outros modelos, tenderá a revelar o status em que a GC se encontra mediante esse paradigma. Diante desse status, a academia tende a vislumbrar, por meio de novas pesquisas, os caminhos que podem ser seguidos para atingir um estágio ideal de apresentar um modelo totalitário no âmbito do conhecimento organizacional.

Para isso, essa pesquisa se subdivide em seções de forma a organizar o discurso aqui apresentado. Além desta introdução, primeiramente a GC holística e suas dimensões são apresentadas (seção 2) e, em momento posterior, os procedimentos metodológicos a serem utilizados para analisar o modelo de Terra (2005) são elucidados (seção 3). Em sequência (seção 4), o referido modelo é analisado (subseção 4.1) e os resultados advindos dessa análise são expostos (subseção 4.2). Diante deste percurso, as conclusões são apresentadas (seção 5) e as referências, que embasam esta pesquisa, são listadas.

\section{A GESTÃO DO CONHECIMENTO HOLÍSTICA E SUAS DIMENSÕES}

Paradigma consiste em um padrão metodológico utilizado para abordar os problemas da ciência (KUHN, 1998). O paradigma holístico é uma abordagem que considera que o todo seja segmentado em partes (VERGARA, 1993; CREMA, 1991). Entretanto, essas partes devem ser analisadas junto ao todo para que se possa regressar a totalidade do fenômeno em estudo, o distinguindo do paradigma reducionista, pois esse segundo analisa as partes de forma isolada do todo (PEREIRA, 2002; MACIEL; SILVA, 2008; CREMA, 2015).

No âmbito da GC, fragmentada em partes como equipe de GC, tecnologia da informação, cultura organizacional etc. (ZIEBA; ZIEBA, 2014; ARIF; SHALHOUB, 2014; KUMAR; SINGH; HALEEM, 2015), o paradigma holístico é uma forma de abordar o gerenciamento do conhecimento por uma perspectiva íntegra. Cada uma dessas dimensões pode ser analisada de forma isolada do todo (paradigma reducionista), no entanto é necessário que a GC articule essas dimensões considerando suas conexões (paradigma holístico), ou seja, considerando a existência de suas dimensões para que se possa gerenciar o conhecimento em sua completude no contexto organizacional. 
A forma de pôr a GC em prática nas organizações advém das propostas de modelos de GC. Um modelo é uma abstração esquemática da realidade que esclarece os elementos e as interfaces entre esses para o gerenciamento do conhecimento (WEBER, 2002; HEISIG, 2009). Existem diversos modelos publicados com o intento de gerir o conhecimento, todavia esses se orientam a uma dimensão específica, acarretando na não consideração de outras (HOLSAPPLE; JOSHI, 1999; RUBENSTEIN-MONTANO et al., 2001; HEISIG, 2009; FTEIMI, 2015). Assim, modelos de GC holísticos devem articular todas as dimensões do gerenciamento do conhecimento de forma a contemplar a GC em sua totalidade, pois as conexões existentes entre essas partes também constituem o todo.

Todavia, por não haver um consenso entre essas partes, mediante a perspectiva de que "[...] 'Só posso compreender um todo se conheço, especificamente, as partes, mas só posso compreender as partes se conhecer o todo'" (MORIN, 2005, p. 181), torna-se necessário consolidar as partes (dimensões) que conformam o gerenciamento do conhecimento, pautado no paradigma holístico. Para isso, a perspectiva de Fatores Críticos de Sucesso se torna viável de ser considerada, já que são as áreas empresariais, se os resultados forem suficientes, que tendem a garantir o desempenho e o sucesso organizacional bem sucedido (ROCKART, 1979). No âmbito da GC, são os fatores críticos que orientam o sucesso do gerenciamento do conhecimento no contexto organizacional.

Uma pesquisa realizada na base SCOPUS, de forma a identificar e consolidar os Fatores Críticos de Sucesso para a GC, resultou na solidificação de 13 áreas críticas para o sucesso do gerenciamento do conhecimento. Essas áreas, também denominadas dimensões, foram obtidas por meio da análise dos estudos de Skyrme e Amidon (1997), Davenport, De Long e Beers (1998), Wong (2005), Wong e Aspinwall (2005), Akhavan, Jafari e Fathian (2006), Al-Mabrouk (2006), Lin e Lin (2006), Gai e Xu (2009), Abbaszadeh, Ebrahimi e Fotouhi (2010), Valmohammadi (2010), Wai, Hong e Din (2011), Sedighi e Zand (2012), Silva Junior, Polizel e Silva (2012), Arif e Shalhoub (2014), Zieba e Zieba (2014) e Kumar, Singh e Haleem (2015). Assim, as dimensões da GC holística são:

a) Estratégia: a estratégia da organização deve ser alinhada à estratégia de GC (AKHAVAN; JAFARI; FATHIAN, 2006), de forma clara e bem definida (WONG; ASPINWALL, 2005; SILVA JUNIOR; POLIZEL; SILVA, 2012) e comunicada aos membros da organização para que tenham compreensão dos objetivos almejados pelo gerenciamento do conhecimento (WONG, 2005; LIN; LIN, 2006; GAI; XU, 2009);

b) Liderança e suporte da alta administração: a liderança pode influenciar o comportamento dos indivíduos (WONG; ASPINWALL, 2005; SILVA JUNIOR; POLIZEL; SILVA, 2012) e sem o apoio de líderes e suporte da alta administração, a GC pode não ser realizada com sucesso (KUMAR; SINGH; HALEEM, 2015). Os líderes, por meio de palavras e exemplos (GAl; XU, 2009; VALMOHAMMADI, 2010; WAl; HONG; DIN, 2011), devem atuar junto à alta administração, concedendo tempo e recursos para a GC (WONG; ASPINWALL, 2005; WONG, 2005; GAl; XU, 2009; ARIF; SHALHOUB, 2014; KUMAR; SINGH; HALEEM, 2015);

c) Equipe de gestão do conhecimento: são as pessoas orientadas a promover a GC (DAVENPORT; DE LONG; BEERS, 1998), atuando com papéis específicos (WONG; ASPINWALL, 2005; VALMOHAMMADI, 2010), responsabilidades (ARIF; SHALHOUB, 2014), definindo processos de coordenação e gestão (GAl; XU, 2009) e o rumo da GC organizacional (WONG, 2005);

d) Recursos (financeiro, humano, material e tempo): a GC demanda suporte financeiro para sistemas tecnológicos específicos (AL-MABROUK, 2006; ABBASZADEH; EBRAHIMI; FOTOUHI, 2010), pessoas para a execução do gerenciamento do conhecimento (WONG, 2005), recursos materiais para a operacionalização do trabalho (KUMAR; SINGH; HALEEM, 2015) e tempo para realizar as atividades propostas em prol do conhecimento (AL-MABROUK, 2006);

e) Processos e atividades: são o centro da GC (SEDIGHI; ZAND, 2012), visto que revelam como o conhecimento será manipulado (WONG, 2005; AKHAVAN; JAFARI; FATHIAN, 2006; ABBASZADEH; EBRAHIMI; FOTOUHI, 2010), sendo criação, armazenamento, identificação e compartilhamento do conhecimento alguns exemplos (WONG, 2005) que devem estar ligados ao trabalho dos indivíduos (GAl; XU, 2009);

f) Gestão de recursos humanos: por atuar junto às pessoas, possuidoras de conhecimento (SEDIGHI; ZAND, 2012), a gestão de recursos humanos é vital para o gerenciamento do conhecimento (VALMOHAMMADI, 2010). Os processos de recrutamento, desenvolvimento e retenção de indivíduos são imperativos para o sucesso da GC (ABBASZADEH; EBRAHIMI; FOTOUHI, 2010);

g) Treinamento e educação: as pessoas devem ser educadas quanto ao vocábulo da GC (DAVENPORT; DE LONG; BEERS, 1998) e treinadas quanto ao uso das ferramentas orientadas ao conhecimento (GAI; XU, 2009), à relevância de compartilhar o conhecimento (VALMOHAMMADI, 2010) e à prática de trabalho colaborativo (SKYRME; AMIDON, 1997; WONG, 2005). Os novos contratados devem ser treinados quanto aos quesitos anteriores, bem como quanto à cultura almejada e a processos organizacionais existentes (KUMAR; SINGH; HALEEM, 2015);

h) Motivação:os incentivos são meios para que os funcionários se envolvam e pratiquem a GC (AL-MABROUK, 2006; LIN; LIN, 2006), sendo uma ferramenta de motivação à prática colaborativa de compartilhamento do conhecimento (WONG, 2005; SEDIGHI; ZAND, 2012). Tais incentivos podem ser 
recompensas financeiras VALMOHAMMADI, 2010), reconhecimento (WONG; ASPINWALL, 2005; SILVA JUNIOR; POLIZEL; SILVA, 2012) e valoração do indivíduo (ZIEBA; ZIEBA, 2014), devendo ser projetados a longo prazo (WONG, 2005; SEDIGHI; ZAND, 2012);

i) Trabalho em equipe: as equipes de trabalho constituem um alicerce benéfico para a criação e compartilhamento do conhecimento (WAl; HONG; DIN, 2011). Assim, o trabalho em equipe tende à cooperação entre as pessoas e ao aumento do sucesso das práticas e processos de GC (SEDIGHI; ZAND, 2012);

j) Cultura: consiste em valores, normas e costumes sociais (AL-MABROUK, 2006; SILVA JUNIOR; POLIZEL; SILVA, 2012). Uma cultura propícia à GC deve prover uma atmosfera voltada ao conhecimento (WONG, 2005) e tende a estabelecer um alto grau de confiança mútua entre os funcionários (GAl; XU, 2009; VALMOHAMMADI, 2010) para um ambiente colaborativo (LIN; LIN, 2006; AL-MABROUK, 2006);

k) Tecnologia da informação: a relevância da tecnologia para a GC é indiscutível (WONG, 2005), pois é um facilitador eficaz de manipulação (captura, armazenamento e divulgação) do conhecimento (ALMABROUK, 2006). Entretanto,essa deve ser percebida como um meio para os processos da GC e não como uma solução definitiva (ABBASZADEH; EBRAHIMI; FOTOUHI, 2010; VALMOHAMMADI, 2010);

I) Mensuração: resulta em determinar metas mensuráveis para posterior aferição (LIN; LIN, 2006), podendo fazer uso de indicadores financeiros e não financeiros (GAl; XU, 2009), pois medir a GC é necessário para que seja possível identificar se os objetivos foram alcançados e avaliar o seu progresso (VALMOHAMMADI, 2010; KUMAR; SINGH; HALEEM, 2015). Empregado por auditorias, os diagnósticos são meios que tendem a medir a GC em diversas faces e identificar lacunas a serem sanadas (AKHAVAN; JAFARI; FATHIAN, 2006);

m) Projeto Piloto: poucas organizações aplicam a GC em toda a instituição (SKYRME; AMIDON, 1997), sendo comum o uso de projetos pilotos como uma forma de aplicar a GC em menor âmbito, visando ao aprendizado e ao aumento do potencial de acerto em investidas de maior amplitude (SKYRME; AMIDON, 1997; AKHAVAN; JAFARI; FATHIAN, 2006).

As 13 dimensões supracitadas constituem a GC holística e, como tal, devem ser consideradas em modelos orientados ao gerenciamento do conhecimento, porquanto são críticas para o sucesso da GC. Assim, essas são os elementos a serem identificados na análise a ser realizada sobre o modelo de Terra (2005). Os procedimentos metodológicos adotados para prover esse exame são expostos na seção seguinte desta pesquisa.

\section{PROCEDIMENTOS METODOLÓGICOS}

Esta pesquisa faz uso da Análise de Conteúdo qualitativa para promover o exame do modelo de GC de Terra (2005). A Análise de Conteúdo, proposta por Bardin (1977), apresenta-se como "Um conjunto de técnicas de análise das comunicações visando obter, por procedimentos, sistemáticos e objectivos de descrição do conteúdo das mensagens, indicadores (quantitativos ou não) que permitam a inferência de conhecimentos relativos às [...] mensagens (BARDIN, 1977, p. 42, itálico original). Em outros termos, resulta em um método que se orienta pelo exame do conteúdo de mensagens para a produção de inferências.

Para aplicação do método, torna-se necessária a determinação das unidades de registros (BARDIN, 1977), elementos a serem investigados no conteúdo analisado, de modo a permitir sua posterior ordenação, categorização e contagem frequencial (BARDIN, 1977). As unidades de registro desta pesquisa são as 13 dimensões da GC holística, delineadas na seção anterior desta pesquisa.

Cientes de que a abordagem qualitativa admite a subjetividade do pesquisador, o exame do modelo de Terra (2005) será conduzido de forma a demonstrar o percurso cognitivo que sustenta as inferências realizadas quanto à presença ou ausência das dimensões da GC holística no modelo. Assim, o rigor científico exposto por Flick (2004), necessário na abordagem qualitativa, é empregado de forma a dar consistência e rigidez às significações apreendidas.

A escolha do modelo de GC de Terra (2005) deve-se ao fato de esse ser uma das referências nacionais mais citadas em GC, segundo resultados dos estudos de Santos et al. (2007) e Corrêa, Ziviani e Chinelato (2016), o que confere credibilidade à obra e suscita o interesse em seu exame. Entretanto, é importante ressaltar que os resultados da análise a serem realizadas sobre o referido modelo não se orientam a conferir mérito ou demérito à obra, mas identificar o quanto essa se adere ao gerenciamento do conhecimento pautado no paradigma holístico.

\section{ANÁLISE E RESULTADOS}

Os procedimentos metodológicos, descritos na seção anterior, são empregados nesta seção e apresentados em dois momentos. Primeiramente (subseção 4.1), a Análise de Conteúdo é aplicada ao modelo de forma a identificar a presença ou ausência das dimensões da GC holística. Em momento 
posterior (subseção 4.2), os resultados, advindos da análise anterior, são quantificados de forma a revelar o grau de adesão do modelo à GC holística. Em continuidade, a seção seguinte apresenta a análise promovida sobre todo o modelo de Terra (2005).

\subsection{Análise do modelo de terra (2005)}

Terra (2005) exprime a sutileza e o intricamento da GC. A sutileza é sustentada pela premissa de que o conhecimento não pode ser gerenciado em si, pois reside na cabeça das pessoas e, portanto, a GC se ocupa "dos processos gerenciais e infra-estrutura física e digital que facilitam, favorecem e estimulam os processos humanos de criação, compartilhamento e uso dos conhecimentos individuais e coletivos" (TERRA, 2005, p. 2). Essa percepção revela que gerir o conhecimento não é gerir os indivíduos que o detêm, ou mesmo o próprio conhecimento inerente a esses, mas promover aspectos organizacionais para que o conhecimento possa ser criado, compartilhado e utilizado na organização.

Pela amplitude dos aspectos organizacionais que circundam a GC, como os supracitados processos organizacionais e infraestrutura, o intricamento entre esses elementos se estabelece. Conforme Terra (2005), é preciso considerar pessoas, estratégia, cultura, tecnologia e processos, simultaneamente, o que confere à GC uma perspectiva transdisciplinar. Juntamente à observância desses elementos, essa temática

exige um certo grau de sofisticação intelectual, empatia verdadeira para envolver os colaboradores e também capacidade de abstração para compreender bem a natureza do conhecimento que se quer gerir e quais ferramentas, métodos e processos são mais eficazes para estimular seu desenvolvimento, compartilhamento e proteção (TERRA, 2005, p. 2).

Mediante à compreensão de que a GC não atua sobre o conhecimento em si, mas sobre elementos que a circundam e apresentam características complicadas quanto as suas interações, Terra (2005) clarifica sua percepção quanto à GC da seguinte forma:

Gestão do Conhecimento significa organizar as principais políticas, processos e ferramentais gerenciais e tecnológicos à luz de uma melhor compreensão dos processos de GERAÇÃO, IDENTIFICAÇÃO, VALIDAÇÃO, DISSEMINAÇAO, COMPARTILHAMENTO, PROTEÇÃO e USO dos conhecimentos estratégicos para gerar resultados (econômicos) para a empresa e benefícios para os colaboradores internos e externos (stakeholders) (TERRA, 2005, p. 8, itálico original).

Os resultados (econômicos), oriundos da GC, podem surgir em curto ou longo prazo, mas não se pode investir no conhecimento considerando seu retorno imediato. Deve-se investir nesse recurso tendo a crença de que esse gera resultados empresariais e, conforme Terra (2005, p. 113), "o investimento no recurso conhecimento envolve, de certa maneira, uma fé em que, quando necessário, ele se manifestará de forma ágil, eficiente e pertinente". O futuro é imprevisível e se preparar para o futuro é acreditar que o conhecimento é um recurso chave. Em suma, as organizações que investem em GC "estão se preparando melhor para o futuro, mas colhendo hoje os resultados dos investimentos passados, pois o investimento é cumulativo" (TERRA, 2005, p. 114).

Envolvido pela perspectiva da GC como algo sutil, complicado, imprevisível quanto aos resultados econômicos a serem obtidos no futuro e amplo no que tange seu conceito, Terra (2005) demarca seu modelo de GC composto por 7 dimensões, sendo: visão e estratégia - alta administração (1), cultura organizacional (2), estrutura organizacional (3), políticas de recursos humanos (4), sistemas de informação (5), mensuração de resultados (6) e ambiente externo (7), conforme Figura 1. 
Ambiente Externo (7)

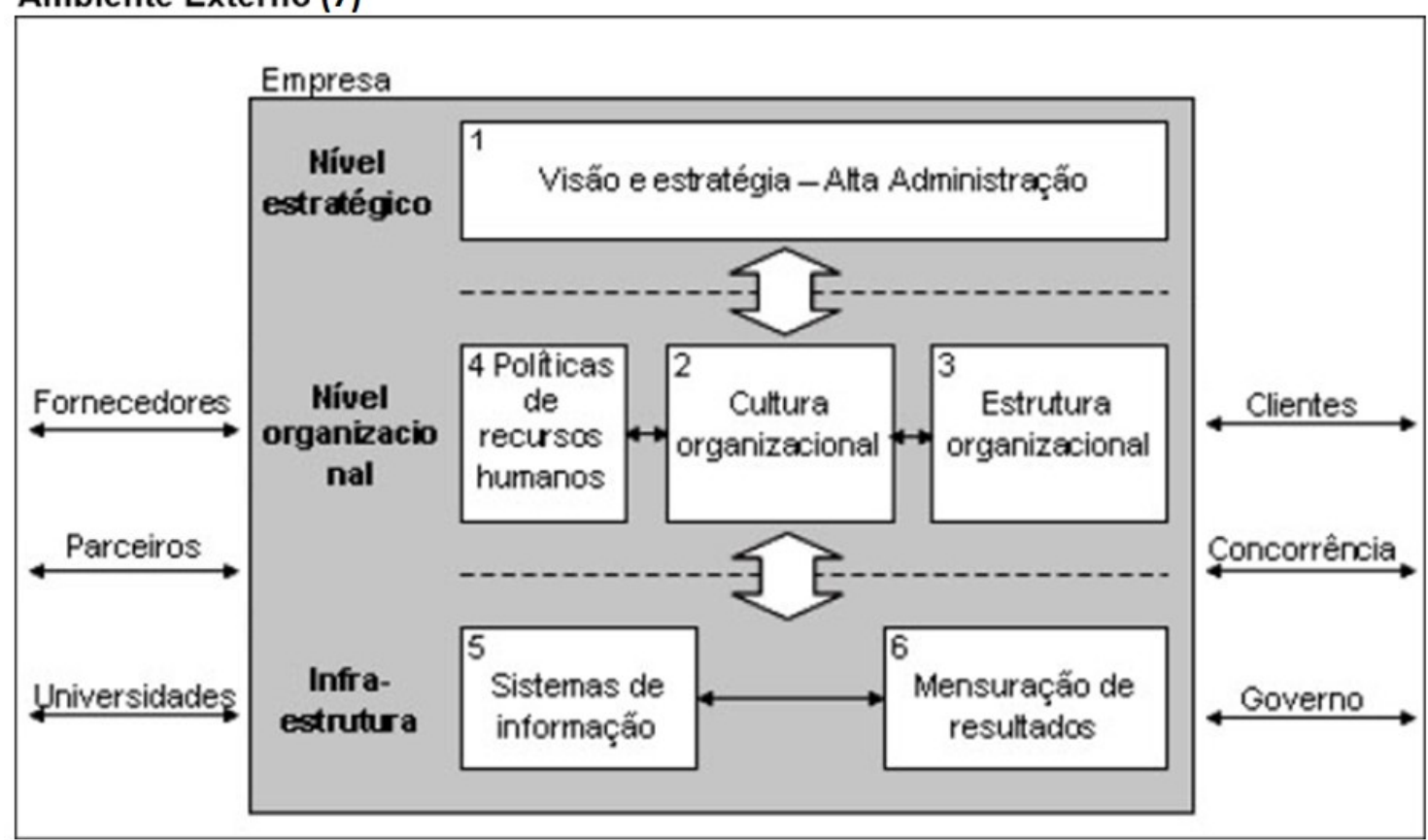

FONTE: Terra (2005, p. 86).

A dimensão visão e estratégia - alta administração (1) posiciona a alta administração como responsável pela "definição dos campos de conhecimento [...] além de seu papel indispensável na clarificação da estratégia empresarial e na definição de metas desafiadoras e motivantes" (TERRA, 2005, p. 86). A definição dos campos de conhecimento visa orientar a empresa e seus funcionários sob quais áreas de conhecimento devem ser conduzidos seus esforços de aprendizado, interligando tais esforços e "as várias atividades individuais a algo maior, a um propósito ou a um destino final" (TERRA, 2005, p. 106).

Terra (2005) sinaliza que as empresas devem determinar suas competências-chave, pois "o fator crítico de êxito empresarial passa a ser a capacidade das empresas em dominar um conjunto limitado de habilidades e áreas de conhecimento que são importantes para seus clientes ou consumidores" (TERRA, 2005, p. 106, sublinho dos autores). 0 autor também atribui à alta administração a incumbência de clarificar a estratégia empresarial de forma a tornar essa entendível aos demais membros da organização.

Ademais, é demarcada a necessidade de estabelecimento de uma estratégia de conhecimento. A ideia é detalhar os conhecimentos necessários para o atingimento da estratégia proposta pela alta administração e, assim, desenvolver as competências essenciais visando a tal atingimento. Conforme Terra (2005):

A elaboração de 'Estratégia de Conhecimento' compartilhada requer, normalmente, a realização de uma série de workshops com as principais lideranças da organização para detalhar a noção de core competencies. Em termos concretos, a Estratégia de Conhecimento se traduz em taxonomias bastante detalhadas associadas a cada uma das prioridades estratégicas da alta administração (TERRA, 2005, p. 115, itálico original, sublinho dos autores).

Assim, a definição das competências-chave da organização e dos campos de conhecimento e o desenvolvimento de uma estratégia de conhecimento e sua clarificação aos demais membros da empresa tendem a promover o alinhamento da GC à estratégia de negócio da empresa. O revelado por Terra (2005) se adere à dimensão Estratégia da GC holística, pois a estratégia de negócio deve estar alinhada à estratégia da GC (AKHAVAN; JAFARI; FATHIAN, 2006), devendo essa ser clara e bem definida (WONG; ASPINWALL, 2005; SILVA JUNIOR; POLIZEL; SILVA, 2012) e compartilhada com os membros da organização, para que compreendam os objetivos a serem atingidos (WONG, 2005; LIN; LIN, 2006; GAl; XU, 2009).

Além do exposto, é incumbência da alta administração definir metas ambiciosas e motivantes e criar um senso de urgência. Por metas ambiciosas, Terra (2005) resgata o exemplo da General Eletric, no qual fora estabelecida a meta da empresa em "ser sempre a $1^{\text {a }}$ ou a $2^{\text {a }}$ colocada em todos os mercados em que atua" (TERRA, 2005, p. 110). Criar um sentimento de urgência é uma forma pontuada por Terra (2005) para que as pessoas sejam direcionadas ao atingimento da meta estabelecida, e o apoio da alta administração é determinante para promover o pensamento estratégico.

As supracitadas percepções do autor são aderentes à dimensão Liderança e suporte da alta administração da GC holística. Conforme Wong e Aspinwall (2005) e Silva Junior, Polizel e Silva (2012), a 
liderança assume papel influenciador no comportamento das pessoas para com a GC, sem uma liderança capaz e suporte da alta administração, a GC pode não ser conduzida com sucesso (KUMAR; SINGH; HALEEM, 2015). Por meio do estabelecimento de metas desafiadoras e do senso de urgência, apresentados por Terra (2005), líderes que compõem alta administração podem promover tal influencia no comportamento dos indivíduos.

O suprassumo da dimensão visão e estratégia - alta administração (1) se consolida, para Terra (2005), na ideia de que a alta administração deve liderar e não mais gerenciar como feito nos moldes tradicionais, o que incide em aceitar erros, alavancar as competências essenciais, incentivar a tomada de decisões, dar exemplos de compartilhamento e produção de conhecimento, dentre outros. As ações e direcionamentos da alta administração afetam todas as demais dimensões da GC e, portanto, a liderança demanda "muito mais arte e sabedoria do que nos 'velhos dias' de Fayol" (TERRA, 2005, p. 116).

A dimensão cultura organizacional (2) possui como elementos as "normas e valores da organização que ajudam a interpretar eventos e avaliar o que é apropriado e inapropriado" (TERRA, 2005, p. 120). A alta administração tem papel de fomentar a cultura pela transmissão das normas e valores aos funcionários, por meio de "atos simbólicos e ações [...] que valorizam e destacam fatos, notícias e pessoas que exemplificam os valores perseguidos" (TERRA, 2005, p. 120). Para Terra (2005) o

desenvolvimento de uma cultura organizacional voltada para a inovação, experimentação, aprendizado contínuo e comprometidas com os resultados de longo prazo e com a otimização de todas as áreas da empresa deve ser uma das preocupações fundamentais da alta administração (TERRA, 2005, p. 86).

Conforme dimensão Liderança e suporte da alta administração da GC holística e corroborando com Terra (2005), os líderes devem atuar como modelos não somente por palavras, mas por meio de atos e exemplos a serem desempenhados pelos demais membros (GAl; XU, 2009; VALMOHAMMADI, 2010; WAI; HONG; DIN, 2011). Devem apoiar a GC em diversos fatores além do financeiro, criando, assim, condições para o desenvolvimento da GC (GAl; XU, 2009) e para um ambiente de criação de conhecimento e aprendizagem (WONG; ASPINWALL, 2005). O compromisso da alta administração contribui, efetivamente, como um sustentáculo que subsidia a proposta da GC (WONG, 2005).

Um aspecto da cultura é a confiança, compreendida pela ética das ações que ocorrem entre as pessoas e entre as pessoas e a empresa, e reflete o ato de compartilhamento do conhecimento, pois as pessoas tendem a compartilharem seus conhecimentos quando confiam na organização ou em outrem (TERRA, 2005). Esse aspecto é aderente à dimensão Cultura da GC holística, pois a cultura consiste em valores, normas e costumes sociais (AL-MABROUK, 2006; SILVA JUNIOR; POLIZEL; SILVA, 2012) e tende a estabelecer um alto grau de confiança mútua entre os funcionários (GAl; XU, 2009; VALMOHAMMADI, 2010) para um ambiente colaborativo (LIN; LIN, 2006; AL-MABROUK, 2006).

O autor demarca que o incentivo ao compartilhamento ocorre por meio de celebrações, nas quais "a alta administração reconhece ações e resultados que estão alinhados com seus valores, metas e objetivos" (TERRA, 2005, p. 137), dentre outras ações como

comunicação direta da alta administração ou gerentes com todos os subordinados, apresentação de desenvolvimentos, novas idéias e inovações ocorridas em determinadas partes da organização; reconhecimento por atitudes altamente colaborativas ou de compartilhamento de conhecimentos etc (TERRA, 2005, p. 127).

As formas de incentivo ao compartilhamento, propostas por Terra (2005), se aderem à dimensão Motivação da GC holística, pois os incentivos são meios utilizados para motivar os indivíduos a compartilharem o que sabem em prol de uma prática colaborativa (WONG, 2005; SEDIGHI; ZAND, 2012). Deve-se considerar que tais incentivos não necessariamente estão relacionados a retornos financeiros, podendo ser por meio de recompensas (VALMOHAMMADI, 2010), reconhecimento (WONG; ASPINWALL, 2005; SILVA JUNIOR; POLIZEL; SILVA, 2012) e valoração do indivíduo (ZIEBA; ZIEBA, 2014).

Outro aspecto pontuado por Terra (2005) para a cultura organizacional é o tempo: um recurso escasso, no entanto imprescindível para a GC. Para o autor, os "gerentes devem aceitar a idéia de que a existência de um tempo para aprender e pensar é um dos principais indicadores da orientação da empresa na direção do conhecimento" (TERRA, 2005, p. 132).

A perspectiva de tempo de Terra (2005) se adere à dimensão Liderança e suporte da alta administração da GC holística, pois, para a consecução das atividades orientadas ao conhecimento, a alta administração deve conceder tempo aos líderes no apoio aos demais funcionários nos processos voltados para o conhecimento (KUMAR; SINGH; HALEEM, 2015) bem como os líderes devem prover tempo aos funcionários para a aquisição de novos conhecimentos (ARIF; SHALHOUB, 2014).

A dimensão estrutura organizacional (3) estabelece a promoção de práticas de "organização do trabalho [...] para superar os limites à inovação, ao aprendizado e à geração de novos conhecimentos, impostos pelas tradicionais estruturas hierárquico-burocráticas [...] baseadas no trabalho em equipes 
multidisciplinares" (TERRA, 2005, p. 86) que possuam autonomia. Segundo Terra (2005), duas alternativas são consideradas para tratarem as atuais estruturas hierárquico-burocráticas: estruturas que as sobrepõem (i) e estruturas que as rompem (ii).

As estruturas que sobrepõem as hierárquicas (i) são propostas por dois vieses. Para Terra (2005), por um lado, as estruturas hierárquico-burocráticas não são cabíveis ao cenário da inovação, imprimem barreiras à criatividade; no entanto promovem a acumulação de conhecimentos devido à burocracia imposta nos processos. Por outro lado, as estruturas de força-tarefa facilitam a criação de conhecimento; no entanto não fomentam sua partilha na organização, pois essas estruturas se findam na conclusão dos projetos para os quais foram criadas. Derivadas de práticas japonesas, como kaizen, just-in-time e qualidade total, as novas estruturas postuladas como aderentes ao cenário do conhecimento são propostas como uma fusão das estruturas burocráticas e de força-tarefa (TERRA, 2005, p. 145), de forma a sobrepor a estrutura hierárquico-burocrática, mas sem rompê-la.

As estruturas que rompem as hierárquico-burocráticas (ii) se fundamentam na agilização e descentralização do processo de tomada de decisão. Consistem em delegar autonomia para que pessoas que tenham mais conhecimentos - como aqueles que possuem contato direto com clientes - tomem decisões ao invés de dependerem de outros para isso. Conforme Terra (2005), essa prática demanda a capacitação e suporte de sistemas de informação para prover a essas pessoas apoio na tomada de decisão. Ademais, o autor salienta que essa prática promove um rompimento drástico na forma como as organizações são organizadas, diferentemente das estruturas que visam sobrepor a hierárquico-burocrática - fusão das estruturas burocrática e força-tarefa - e, portanto, se apresentam como desafios maiores que em outros fatores, tais como mudanças culturais e políticas de recursos humanos.

Terra (2005) pondera sobre práticas com foco na aprendizagem organizacional. Diferente das estruturas anteriores que, de forma ampla, visam remover barreiras à inovação - estrutura de sobreposição - ou agilizar a tomada de decisão - estrutura de rompimento -, as formas anunciadas visam acelerar o aprendizado individual e coletivo e não são ponderadas como uma nova estrutura, mas como práticas passíveis de serem adotadas em prol da aprendizagem. A máxima é de que as pessoas aprendem ao longo de suas atividades; no entanto, seus conhecimentos ficam delimitados ao indivíduo ou a áreas organizacionais. Assim, formas como melhores práticas, lições aprendidas, after-actionreviews, lunch-andlearn, knowledge-based systems e comunidades de prática são práticas mencionadas que visam à aprendizagem individual e coletiva (TERRA, 2005).

Grande parte das instituições, sejam elas pequenas ou médias, privadas ou públicas, sejam industrial ou de serviços, se estabeleceram sobre os preceitos burocráticos. As alternativas supracitadas são expressas como meios que visam, de alguma forma, superar o modelo clássico hierárquico-burocrático, reconhecido como não aderente para enfrentar os desafios de uma economia dinâmica de conhecimento (TERRA, 2005). Embora tais estruturas e práticas tenham sido explanadas por Terra (2005), o autor não sugere, pondera ou sinaliza a adoção de alguma das estruturas - sobreposição ou rompimento da hierárquico-burocrática - ou práticas de aprendizagem individual e coletiva em seu modelo.

A dimensão denominada políticas de recursos humanos (4) aborda práticas "associadas à aquisição de conhecimentos [pessoas] externos e internos à empresa, assim como à geração, difusão e armazenamento de conhecimento na empresa" (TERRA, 2005, p. 86). Para Terra (2005), as pessoas, além da remuneração financeira inerente ao labor desempenhado, procuram o desenvolvimento pessoal e são mais leais a sua profissão do que à empresa em que trabalham. A empresa, por sua vez, visa elevar seu estoque de conhecimento e, necessariamente, depende das pessoas, pois essas o detêm. Diante desse cenário, o autor pondera sobre os aspectos recrutamento e seleção (i), treinamento (ii), carreira (iii) e sistemas de avaliação de recompensa (iv), relacionados às políticas de recursos humanos (4).

Essa dimensão do modelo de Terra (2005) corresponde à dimensão Gestão de recursos humanosda GC holística. A gestão de recursos humanos é imperativa para tratativas relacionadas ao conhecimento, pois as pessoas são a pedra angular da GC (VALMOHAMMADI, 2010), por serem essas as detentoras do conhecimento (SEDIGHI; ZAND, 2012). Portanto, os processos de recrutamento, desenvolvimento e retenção de funcionários, desempenhados pela gestão de recursos humanos, são vitais para o sucesso da GC (ABBASZADEH; EBRAHIMI; FOTOUHI, 2010).

Segundo Terra (2005), o recrutamento e seleção (i) é um dos pontos mais importantes para atrair. Isso se dá pelo fato de que o perfil esperado para a empresa envolve características como qualificação, criatividade, capacidade de trabalhar em grupo e iniciativa. Portanto, um funcionário pode aumentar ou diminuir as competências e outros ativos intangíveis da organização em detrimento da existência dessas características em seu perfil.

Assim, os processos de seleção de pessoal são "cada vez mais intensos e competitivos e [...] exigem grande dedicação de tempo e recursos, inclusive da alta administração" (TERRA, 2005, p. 163). Por intenso se tem o rigor na seleção pela busca de perfis com características adequadas à empresa. Competitivos por que a relação de candidatos por vaga oscila entre 50 a 100. Quanto aos recursos, a Toyota exemplifica a 
quantia de US\$ 3 mil por candidato, aproximadamente, no processo de seleção (TERRA, 2005). Em essência, Terra (2005) demonstra a importância desse aspecto para as empresas que buscam funcionários com perfil aderentes à era do conhecimento.

O treinamento (ii) resgata a capacitação nos moldes paternalistas (empresa delega ao empregado). No entanto, Terra (2005, p. 168) reflete que as empresas "já não conseguem determinar, centralmente, quais são todas as habilidades e competências que cada área ou funcionário precisa desenvolver" e, portanto, o modelo paternalista parece estar se movendo para o modelo voltado ao a prendizado. Nesse novo modelo, "cabe às empresas fornecer os recursos adequados (tempo e dinheiro) e criar ambientes que estimulem e promovam valores relacionados ao crescimento pessoal e ao treinamento on-the-job" (TERRA, 2005, p. 168, itálico original, sublinho dos autores). Conforme dimensão Recursos (financeiro, humano, material e tempo) da GC holística, o gerenciamento do conhecimento demanda aporte financeiro (AL-MABROUK, 2006; ABBASZADEH; EBRAHIMI; FOTOUHI, 2010).

Duas vertentes são consideradas. A primeira é a ideia de que o funcionário busque aprender no desempenho de sua função e ensine aos outros de forma continua. $O$ aprendizado passa a ser pelo repasse de conhecimentos dos mais experientes para os menos experientes, acarretando em benefícios mútuos, pois quem ensina também aprende. Terra (2005) menciona a abordagem da Microsoft que, por um lado, busca pessoas que aprendam por conta própria e, por outro, os apoia, apontando indivíduos mais experientes que atuam como mentores, assumindo, também, a responsabilidade de ensinar além de suas atividades cotidianas.

A segunda vertente considera que a empresa pode subsidiar a capacitação, por meio de treinamento, e-learning, cursos em universidades, feiras e congressos, como ocorre na Xerox do Brasil. Em ambas as vertentes, o funcionário deve buscar o próprio treinamento, cabendo à empresa apoiá-lo no que for atinente às necessidades da organização (TERRA, 2005).

A carreira (iii) é outro aspecto relacionado às políticas de recursos humanos (4). $O$ discurso de Terra (2005) é pautado na movimentação dos funcionários na empresa. Essa movimentação permite que o funcionário experiencie diversas áreas e funções da empresa, o que eleva sua qualificação e visão acerca da empresa, aumenta sua capacidade inovadora e o torna um veículo de troca de informações, devido ao estabelecimento de diferentes relações em detrimento da sua movimentação (TERRA, 2005).

Segundo Terra (2005), os sistemas de avaliação de recompensa (iv) apresentam inclinação para que se passe a considerar a cooperação, oriunda do trabalho em equipe, como uma forma de avaliação de desempenho e de recompensa, não sendo mais condizente avaliar o desempenho sob a perspectiva individual. Embora essa inclinação seja percebida na literatura, para Terra (2005), na prática, "a grande maioria das empresas ainda estaria adotando apenas os esquemas associados, exclusivamente, ao desempenho individual" (TERRA, 2005, p. 179).

Uma tendência de avaliação é o pagamento por competências, no qual é considerada a capacidade de entrega do indivíduo ao invés de seu cargo associado ao organograma da empresa. O pagamento pode considerar desde os clássicos salários até ações da empresa e participação nos lucros, podendo, inclusive, contemplar o compartilhamento do conhecimento no sistema de avaliação (TERRA, 2005). No entanto, Terra (2005) observa que as organizações ainda têm o cargo como o componente central do processo de avaliação. Isso pode ser compreendido por ser uma forma mais fácil de se promover as avaliações na empresa ou pelo costume de determinação de cargos, imbricado nas organizações, oriundo de longos anos do paradigma industrial.

O sistema de avaliação de recompensa (iv), exposto por Terra (2005), adere à Dimensão Motivação da GC holística. Os incentivos projetados a longo prazo servem para apoiar o sistema de avaliação de desempenho dos funcionários (WONG, 2005; SEDIGHI; ZAND, 2012) e consiste no estabelecimento de formas de recompensas para que os funcionários se envolvam e pratiquem a GC (AL-MABROUK, 2006; LIN; LIN, 2006). Pode fazer uso de recompensas (VALMOHAMMADI, 2010), reconhecimento (WONG; ASPINWALL, 2005; SILVA JUNIOR; POLIZEL; SILVA, 2012) e formas de valoração do indivíduo (ZIEBA; ZIEBA, 2014).

Terra (2005) discorre sobre diversas tendências quanto aos aspectos de seleção (i), treinamento (ii), carreira (iii) e sistemas de avaliação de recompensa (iv). No que tange a esse último, são expostas diversas óticas relacionadas ao sistema de avaliação e a formas de recompensa (financeiras e não financeiras). No entanto, o autor não assinala as passíveis de operacionalização em relação ao seu modelo, bem como não sugere a adoção de alguma prática relacionada à seleção (i) e à carreira (iii).

Em relação ao treinamento (ii), Terra (2005) assinala que, por um lado, espera-se que o funcionário busque aprender no desempenho de sua função e ensine aos outros de forma continua. Por outro lado, a empresa pode subsidiar a capacitação, por meio de treinamento, e-learning, cursos em universidades, feiras e congressos. Ambos as vertentes se relacionam com a dimensão Treinamento e educação da GC holística, pois municiam profissionais de conhecimentos que possam ser úteis em suas atividades e permitem melhor engajamento do indivíduo na organização (KUMAR; SINGH; HALEEM, 2015). 
A dimensão sistemas de informação (5) aponta, por um lado, a utilidade de sistemas como datamining para encontrar conhecimento em meio a elevadas quantidades de dados; por outro lado, os sistemas podem ser aplicados para promover o compartilhamento de informação e conhecimento na organização (TERRA, 2005). É indiscutível que os "avanços na informática, nas tecnologias de comunicação e nos sistemas de informação estão afetando os processos de geração, difusão e armazenamento de conhecimento nas organizações" (TERRA, 2005, p. 86, itálico original) e apresentam novas possibilidades à GC, mas o contato pessoal permanece como essencial às organizações.

Terra (2005) demarca que investimentos em tecnologia, voltados para promover o compartilhamento de informação e conhecimento, não garantem o aprendizado. Isso porque conhecimento é fruto do ato interpretativo do indivíduo e sua transferência não criva o aumento de competência ou conhecimento. A máxima é que "não existe gestão do conhecimento de maneira efetiva se não houver aprendizado individual e organizacional" (TERRA, 2005, p. 188).

Esses elementos se aderem à dimensão Tecnologia da informação da GC holística, pois a tecnologia da informação é um facilitador eficaz de manipulação (captura, armazenamento e divulgação) do conhecimento (AL-MABROUK, 2006) e sua relevância para a GC é indiscutível (WONG, 2005); no entanto, é imperativo frisar que essa deve ser percebida como um meio para os processos da GC e não como uma solução definitiva (ABBASZADEH; EBRAHIMI; FOTOUHI, 2010; VALMOHAMMADI, 2010).

A dimensão mensuração de resultados (6) busca a mensuração "sob várias perspectivas e [...] sua comunicação por toda a organização" (TERRA, 2005, p. 86), reconhecendo a contribuição dos estudos de capital intelectual quanto à avaliação das dimensões da GC. O capital intelectual busca medir os ativos intangíveis da empresa e, diferentemente dos tradicionais sistemas contábeis tangíveis que remontam 500 anos e mensuram por análise quantitativa com referência de valor no passado (ativos tangíveis = presente passado), o capital intelectual apresenta indicadores mais qualitativos e tem por referência de valor o futuro, o que o torna difícil de medir (TERRA, 2005).

Embora a mensuração dos ativos intangíveis seja um desafio, esse é um campo de estudo no qual esforços são empregados. Dentre os apontados por Terra (2005), destacam-se o relatório de 1994 da empresa sueca Skandia (EDVINSSON; MALONE, 1998) e a obra de Sveiby (1998) como os pioneiros. Terra (2005) discorre sobre o balanced scorecard (KAPLAN; NORTON, 1997) e pondera sobre a obra Mapas estratégicos (KAPLAN; NORTON, 2004) dos mesmos autores, mencionando que a dimensão de aprendizado e crescimento estava presente na primeira e se tornou foco na segunda obra. Entretanto, Terra (2005) é rígido ao criticar o segundo livro.

Nossa análise, tanto dos exemplos e conceitos utilizados no último livro dos autores, como de casos reais de empresas brasileiras, nos leva a conclusão de que realmente "Aprendizado e Crescimento" são de fato o lado fraco do Balanced Scorecard. Não precisa ser muito cético para ver que há muito "chute" nos próprios exemplos utilizados pelos autores como, por exemplo, um objetivo de $75 \%$ de "best pratice sharing" ou $95 \%$ de "information portfolio readiness" ou ainda um determinado percentual de funcionários no "Clube do Presidente" (TERRA, 2005, p. 224).

Para Terra (2005, p. 225), a obra Mapas estratégicos "ainda deixa muito a desejar no contexto da mensuração de resultados na dimensão Aprendizado e Crescimento [...] o reducionismo aplicado à dimensão conhecimento leva a um falso conforto", corroborando com a percepção de Probst, Raub e Romhardt (2002) de que, embora o balanced scorecard possua o aprendizado como um aspecto de medição, esse "não inclui um método para operacionalizar a dimensão do conhecimento, nem oferece indicadores de conhecimento" (PROBST; RAUB; ROMHARDT, 2002, p. 201).

Por conseguinte, Terra (2005) assegura a necessidade de mensuração e afirma que medidas considerando o conhecimento podem e devem se valer de vários indicadores, mas "jamais teremos algo tão preciso e unificador como muitos chegam a afirmar. Trata-se da natureza do que se busca medir" (TERRA, 2005, p. 226).O autor também assinala que "não se deve confundir [...] Mensuração do Capital intelectual com Gestão do Conhecimento" (TERRA, 2005, p. 228), pois o primeiro visa à valoração dos ativos intangíveis, enquanto o segundo se volta para o aprendizado, geração do conhecimento e gestão dos ativos intangíveis.

A supracitada dimensão mensuração de resultados (6) se adere diretamente à dimensão Mensuração da GC holística, pois medir a GC é necessário para que seja possível identificar se os objetivos foram alcançados e avaliar o seu progresso (VALMOHAMMADI, 2010; KUMAR; SINGH; HALEEM, 2015). Resulta em determinar metas mensuráveis para posterior medição (LIN; LIN, 2006), sendo comuns indicadores e métricas financeiras e aceitáveis medições não financeiras (GAl; XU, 2009), devido à intangibilidade do conhecimento.

Ademais, Terra (2005, p. 276) apresenta um "questionário sobre gestão do conhecimento" que adere à dimensão Mensuração da GC holística, já que é uma forma empregada pelas auditorias e tendem a medir a GC em diversas faces e identificar lacunas a serem sanadas (AKHAVAN; JAFARI; FATHIAN, 2006). 
A última dimensão do modelo de Terra (2005) é denominada ambiente externo (7) e tem por intento engajar "processos de aprendizagem com o ambiente, em particular por meio de alianças com outras empresas e do estreitamento do relacionamento com clientes" (TERRA, 2005, p. 86). Os clusters empresariais são uma forma na qual as empresas atuam colaborativamente e aprendem com os demais atores constituintes dessa estrutura, por meio da troca de intangíveis (conhecimento). As alianças empresariais (join-ventures, acordos de P\&D e troca de tecnologias, associações de pesquisas e outros) também são pontuadas como uma forma colaborativa de promover a cooperação com vistas ao conhecimento (TERRA, 2005).

Os clientes são outra forma de aprendizagem com o ambiente externo, haja vista que "a maior parte das ideias para a inovação se origina da sugestão de clientes" (TERRA, 2005, p. 239). Em alguns casos, o cliente e o fornecedor podem assumir um lugar do outro e, sob esse prisma, o estabelecimento de redes com fornecedores tende a permitir que a empresa aprenda com aqueles que fazem parte de seu negócio e que estão além de suas fronteiras (TERRA, 2005). Em continuidade, o benchmarking é uma ferramenta que permite aprender com o ambiente por meio da comparação de práticas da empresa para com as melhores práticas do mercado (TERRA, 2005), enquanto a inteligência competitiva permite que a empresa aprenda com o ambiente que a circunda por meio do monitoramento desse, visando a um "posicionamento estratégico proativo" (TERRA, 2005, p. 246).

Terra (2005) evidencia a importância de se aprender com o ambiente externo (7), apontando os clusters, alianças, clientes, fornecedores, benchmarking e inteligência competitiva como práticas existentes em outras empresas. Essa relevante contribuição é disposta de forma descritiva, sem que o autor sugira a adoção de uma ou várias ou todas as vertentes em seu modelo.

Por fim, nas considerações e reflexões finais Terra (2005), registra o quão comum tem se tornado a formalização da GC nas empresas, por meio da designação de cargos específicos, como o de gerente do conhecimento, que assume os desafios da GC. Relatos de empresas que adotaram práticas de GC anunciam a necessidade de "significativos esforços de conscientização e de comunicação" (TERRA, 2005, p. 268). É também apontado a importância do apoio ativo da alta administração no que tange ao desenvolvimento estratégico e organizacional, investimentos em infraestrutura tecnológica e cultura organizacional.

\subsection{Resultados}

De forma ampla, o modelo de Terra (2005) discorre sobre a GC, demonstrando a relevância de cada dimensão para com o conhecimento. A relação entre as dimensões é determinante na proposta do autor. A exemplo, a tecnologia é pontuada na dimensão estrutura organizacional (3) quando Terra (2005) aborda as estruturas que rompem as hierárquico-burocráticas (ii), assinalando o necessário suporte de sistemas de informação para apoiar o processo de tomada de decisões de pessoas que tenham recebido autonomia para isso. A alta administração é outro fator presente nas dimensões visão e estratégia - alta administração (1) e cultura organizacional (2), também fortemente evidenciado por Terra (2005) no que tange ao seu apoio ativo em diversos contextos da GC. Por síntese, o modelo de Terra (2005) se adere às seguintes dimensões da GC holística.

a) Estratégia: sim. Apresentada como uma dimensão do modelo;

b) Liderança e suporte da alta administração: sim. Apresentada como uma dimensão do modelo;

c) Equipe de gestão do conhecimento: não especificado. É pontuado o quão comum se tem tornado a formalização da GC nas empresas, por meio da designação de cargos específicos, como o de gerente do conhecimento, mas essa dimensão não é contemplada no modelo do autor;

d) Recursos (financeiro, humano, material e tempo): sim. O tempo e dinheiro (financeiro) são pontuados como imperativos para o treinamento das pessoas na gestão de recursos humanos do modelo;

e) Processos e atividades: não especificado. Embora o autor apresente os processos de "geração, identificação, validação, disseminação, compartilhamento, proteção e uso do conhecimento" (TERRA, 2005, p. 8) no conceito de GC, esses não estão presentes na descrição do modelo;

f) Gestão de recursos humanos: sim. Apresentada como uma dimensão do modelo;

g) Treinamento e educação: sim;.

h) Motivação: sim;

i) Trabalho em equipe: não especificado. Reflete sobre a importância desse perfil para as empresas sem ênfase no que tange ao modelo;

j) Cultura: sim. Apresentada como uma dimensão do modelo;

k) Tecnologiada informação: sim. Apresentada como uma dimensão do modelo, denominada sistemas de informação;

I) Mensuração: sim. Apresentada como uma dimensão do modelo;

m) Projeto Piloto: não especificado. 
Mediante à análise promovida sobre o modelo de Terra (2005), as dimensões, acima ponderadas, são dispostas no Gráfico 1, que exprime a adesão do referido modelo as dimensões da GC holística, concernentes a essa pesquisa. A presença de uma dimensão a posiciona na escala 1 (extremidade externa) do gráfico e sua ausência a assinala na escala 0 (centro).

GRÁFICO 1 - Adesão do modelo de Terra (2005) às dimensões da gestão do conhecimento holística

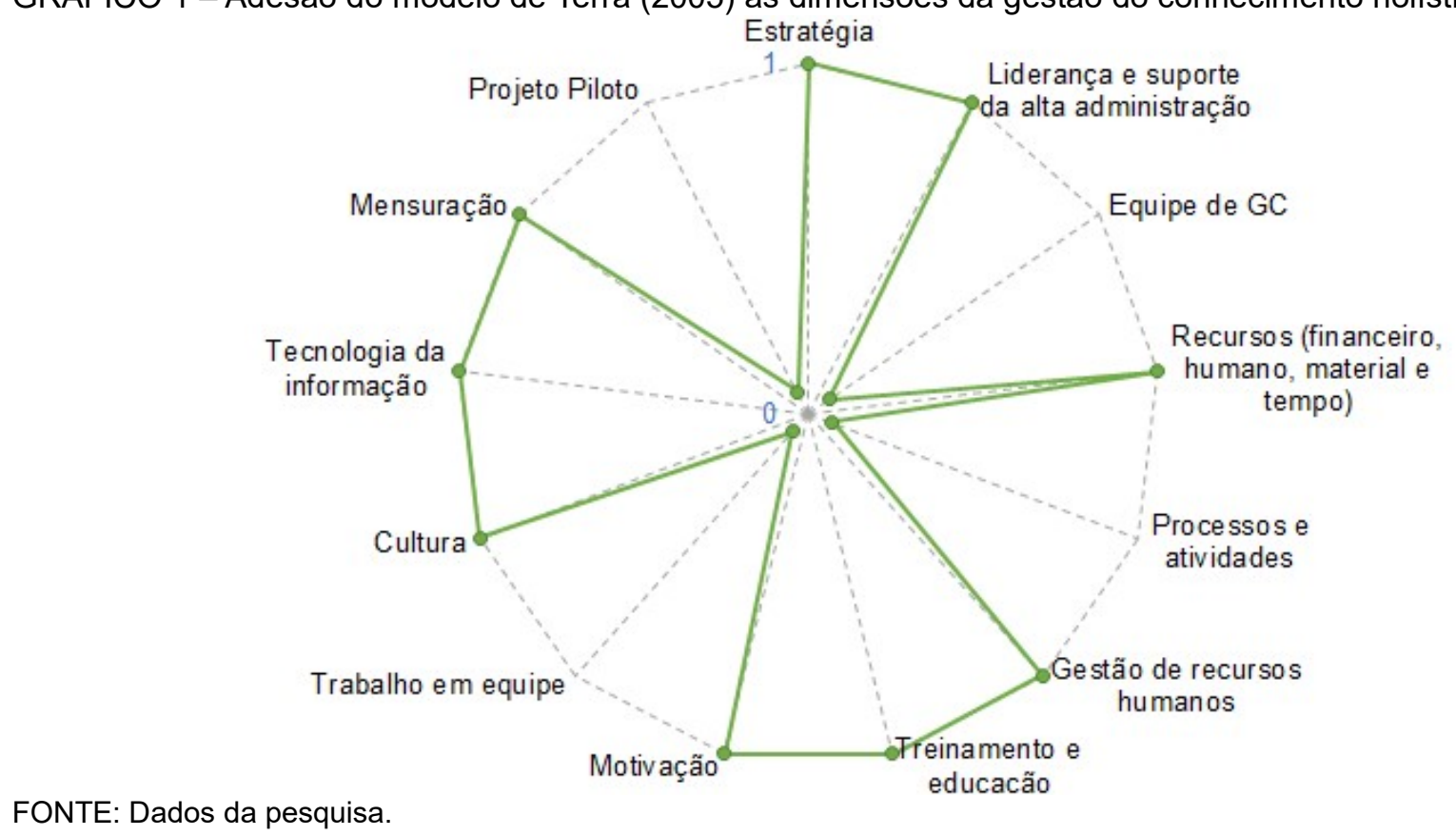

FONTE: Dados da pesquisa.

Assim, o modelo de Terra (2005) contempla as dimensões Estratégia, Liderança e suporte da alta administração, Recursos (financeiro, humano, material e tempo), Gestão de recursos humanos, Treinamento e educação, Motivação, Cultura, Tecnologia da informação e Mensuração, o remetendo ao percentual de $69,2 \%$ (9 do total de 13 dimensões) de adesão ao que considera por GC holística, conforme caracterização apresentada nessa pesquisa.

\section{CONCLUSÃO}

Esta pesquisa apresentou a seguinte questão problema: O modelo de Terra (2005) apresenta aderência a um modelo de GC holístico? Especificamente, este estudo teve por objetivo analisar a aderência do modelo deTerra (2005) a GC holística. Após delinear o gerenciamento do conhecimento, pautado no paradigma holístico, e as dimensões que o compõem, o referido modelo foi analisado integralmente. Por resultado, o modelo de Terra (2005) se adere em $69,2 \%$ pontos percentílicos à GC holística, conforme caracterização apresentada nesta pesquisa, demonstrando haver oportunidade de desenvolvimento de modelos mais abrangentes, haja vista que o modelo analisado não apresenta aderência íntegra à GC holística.

A GC demanda de modelos que se orientem ao todo do gerenciamento do conhecimento, de forma a permitir uma abordagem mais abrangente para as organizações. Por essa perspectiva, esse estudo tende a contribuir com a academia por delinear o que consiste o paradigma holístico para a GC e descrever suas dimensões. Assim, os resultados desta pesquisa tendem a permitir que novos modelos sejam desenvolvidos, considerando todos os aspectos apresentados. Em continuidade, também contribui por fornecer um procedimento de análise de modelos que, se empregada em outros estudos, permitirá que se tenha uma visão totalitária do quanto a GC tem caminhado para uma abordagem ampla nas organizações.

No contexto empresarial, este estudo contribui por apontar as dimensões da GC a serem consideradas nas aplicações práticas do gerenciamento do conhecimento em âmbito organizacional. A análise do modelo de Terra (2005) permite que as empresas tenham o entendimento das potencialidades do modelo e que busquem, em outros estudos, as dimensões não identificadas na obra, permitindo a constituição de um modelo organizacional e sua aplicação prática de forma holística na organização.

Todavia, essa pesquisa se limita por prover a análise de um único modelo. Essa limitação traz consigo a possibilidade de pesquisas futuras que apliquem a análise, aqui apresentada, em outros modelos. Isso tende a demonstrar, como posto anteriormente, o percurso galgado pela GC e orientar novos pesquisadores quanto à necessidade de modelos mais abrangentes, que abordem o todo, ou seja, a integralidade do gerenciamento do conhecimento para sua aplicação prática nas organizações. 


\section{REFERÊNCIAS}

ABBASZADEH, M. A.; EBRAHIMI, M.; FOTOUHI, H.. Developing a causal model of critical success factors for knowledge management implementation. In: International Conference on Education and Management Technology. Anais... ICEMT 2010, p. 701-705, 2010.

AKHAVAN, P.; JAFARI, M.; FATHIAN, M. Critical success factors of knowledge management systems: A multi-case analysis. European Business Review, v.18, n. 2, p. 97-113, 2006.

AL-MABROUK, K. Critical success factors affecting knowledge management adoption: A review of the literature. Anais... 2006 Innovations in Information Technology, IITFALSO, 2006.

ALVES, A. C. et al. Capacidades dinâmicas e de inovação da empresa: Definindo um modelo de avaliação. RAE Revista de Administração de Empresas, v. 57, n. 3, p. 232-244, 2017.

ARIF, M. J.; SHALHOUB, M. H. B. Critical success factors with its effective role in knowledge management initiatives in public and private organizations in saudi Arabia: experts perspectives. Life Science Journal, v. 11, n. 6, p. 636-645, 2014.

BARDIN, L. Análise de conteúdo. Lisboa: Edições 70, 1977.

CORREAA, F. et al. Equalização de tipos de conhecimento em modelos de gestão do conhecimento. In: Encontro Nacional de Pesquisa em Ciência da Informação (EnANCIB), XVIII., Rio de Janeiro, 2007. Anais... Marília: EnANCIB, 2017.

CORREA, F.; ZIVIANI, F.; CHINELATO, F. B. Gestão do conhecimento: uma análise metabibliométrica. Perspectivas em Gestão \& Conhecimento, v. 6, n. 2, p. 208-224, 2016.

CREMA, R. Abordagem holística: integração do método analítico e sintético. In: BRANDÃO, D.; CREMA, R. (orgs). O novo paradigma holístico: ciência, filosofia, arte e mística. São Paulo: Summus, p. 83-99, 1991.

CREMA, R. Introdução à visão holística: breve relato de viagem do velho ao novo paradigma. 6 ed. São Paulo: Summus, 2015 [livro eletrônico].

DAVENPORT, T. H.; DE LONG, D. W.; BEERS, M. C. Successfull knowledge management projects. Sloan Management Review, v. 39, n. 2, p. 43-57, 1998.

FLICK, U. Uma introdução à pesquisa qualitativa. 2. ed. Porto Alegre: Bookman, 2004.

FTEIMI, N. Analyzing the literature on knowledge management frameworks: Towards a normative knowledge management classification schema. In: 23rd European Conference on Information Systems, v. 2015 - May, 2015. Anais... 23rd ECIS 2015, 2015.

GAI, S.; XU, C. Research of critical success factors for implementing knowledge management in China. Anais... 2009 International Conference on Information Management, Innovation Management and Industrial Engineering, ICIII 2009, v. 4, p. $561-564,2009$.

GONCALVES, A. et al. CAPACIDADE DE APRENDIZAGEM ORGANIZACIONAL: ESTUDO REALIZADO EM UMA INDÚSTRIA TÊXTIL.Revista Eletrônica de Estratégia \& Negócios, v. 9, n. 2, p. 166-191, 2016.

GRANT, K A. Tacit knowledge revisited - we can still learn from Polanyi. The Electronic Journal of Knowledge Management, v. 5, n. 2, p. 173-180, 2007.

HEISIG, P. Harmonisation of knowledge management - comparing $160 \mathrm{KM}$ frameworks around the globe. Journal of knowledge management, v. 13, n. 4, p. 4-31, 2009.

HOLSAPPLE, C. W.; JOSHI, K. D. Description and analysis of existing knowledge management frameworks. In: Systems Sciences, 1999. HICSS-32. Anais... 32nd Annual Hawaii International Conference on. IEEE, p. 15, 1999.

KUHN, T. S. A estrutura das revoluções científicas. 5ed. São Paulo: Editora Perspectiva, 1998.

KUMAR, S.; SINGH, V.; HALEEM, A. Critical success factors of knowledge management: modelling and comparison using various techniques. International Journal of Industrial and Systems Engineering, v. 21, n. 2, p. 180-206, 2015.

LEITE, A. A. V.; AGNOLIN, C. T.; CARVALHO, C. E. Fusão entre ALL - América Latina Logística e Rumo Logística Operadora Multimodal: uma análise a partir da Visão Baseada em Recursos. Revista Inteligência Competitiva, v. 7, n. 1, p. 91-128, 2017.

LIN, Y-C.; LIN, L-K. Critical success factors for knowledge management studies in construction. 2006. Anais... Proceedings of the 23rd International Symposium on Robotics and Automation in Construction, ISARC 2006, p. 768-772, 2006.

MACIEL, C.; SILVA, A. Gerenciando pessoas utilizando modelos holísticos. Revista da Administração Contemporânea - RAC, v. 12, n. 1, p. 35-58, 2008.

MORIN, E. Ciência com consciência. 8. ed. Rio de Janeiro: Bertrand Brasil, 2005.

NICKOLS, F. The knowledge in knowledge management. In: WOODS, J. A.; CORTADA, J. The Knowledge Management Yearbook 2000-2001. Butterworth-Heinemann: Boston, p. 12-21, 2000.

PEREIRA, M. F. A gestão organizacional em busca do comportamento holístico. In: ANGELONI, M. T. (org.). Organizações do conhecimento: infra-estrutura, pessoas e tecnologia. São Paulo: Saraiva, p. 2-28, 2002.

PROBST, G.; RAUB, S., ROMHARDT, K. Gestão do conhecimento: os elementos construtivos do sucesso. Porto Alegre: Bookman, 2002. 
RIBEIRO, J. S. A. N. et al. Gestão do conhecimento e desempenho organizacional: integração dinâmica entre competências e recursos, Perspectivas em Gestão \& Conhecimento, v. 7, n. 1, p. 4-17, 2017.

ROCKART, J. F. Chief executives define their own data needs, Harvard Business Review, v. 57, n. 2, p. 81-93, 1979.

RUBENSTEIN-MONTANO, B. et al. A systems thinking framework for knowledge management. Decision support systems, v. 31, n. 1, p. 5-16, 2001.

SANTOS, J. L. S. et al. Mapeamento da produção acadêmica em gestão do conhecimento no âmbito do EnAnpad: uma análise de 2000 a 2006. In: Encontro da Associação Nacional dos Programas de Pós-Graduação em Administração (EnANPAD), 31., Rio de Janeiro, 2007. Anais... Rio de Janeiro: ANPAD, 2007.

SEDIGHI, M.; ZAND, F. Knowledge management: Review of the Critical Success Factors and development of a conceptual classification model. Anais... International Conference on ICT and Knowledge Engineering, p. 1-9, 2012.

SENGE, P. M. A quinta disciplina: arte, teoria e prática da organização de aprendizagem. Best Seller, 1990.

SILVA JUNIOR, A.; POLIZEL, C. E. G.; SILVA, P. O. M. Critical success factors for knowledge management at a private higher education school. Revista Brasileira de Gestao de Negocios, v.14, n. 42, p. 102-122, 2012.

SKYRME, D.; AMIDON, D. The knowledge agenda. Journal of Knowledge Management, v.1, n. 1, p. 27-37, 1997.

SOUSA, C. V.; PRATA, F. S.; PEREIRA, J. R. Gestão do conhecimento como fonte de vantagem competitiva em uma paraestatal mineira. Perspectivas em Ciência da Informação, v. 23, n. 2, p. 154-173, 2018.

TEECE, D. J.; PISANO, G.; SHUEN, A. Dynamic capabilities and strategic management. Strategic Management Journal, v.18, n.7, p. 509-533, 1997.

TERRA, J. C. C. Gestão do conhecimento: o grande desafio empresarial. 5ed. Rio de Janeiro: Elsevier, 2005.

VALENTIM, M. L. P. Gestão da informação e gestão do conhecimento em ambientes organizacionais: conceitos e compreensões. Tendências da Pesquisa Brasileira em Ciência da Informação, v. 1, n. 1, 2014.

VALMOHAMMADI C. Investigation and assessment of critical success factors of knowledge management implementation in Iranian small-to-medium sized enterprises. Journal of Applied Sciences, v.10, n. 19, p. 2290-2296, 2010.

VERGARA, S. C. Sobre a intuição na tomada de decisão. Cadernos EBAP, n. 62, 1993.

WAI, Y. M.; HONG, A. N. H.; DIN, S. B. Critical success factors and perceived benefits of knowledge management implementation: Towards a conceptual framework. Australian Journal of Basic and Applied Sciences, v. 5, n. 10, p. 754-760, 2011.

WEBER, F. et al. Standardisation in knowledge management - towards a common KM framework in Europe. Anais... Proceedings of UNICOM Seminar "Towards Common Approaches \& Standards in KM. 2002.

WERNERFELT, B. A resource-based view of the firm. Strategic Management Journal, v. 5, n.2, p. 171-180, 1984.

WONG, K. Y. Critical success factors for implementing knowledge management in small and medium enterprises. Industrial Management \& Data Systems, v. 105, n. 3, p. 261-279, 2005.

WONG, K. Y; ASPINWALL, E. An empirical study of the important factors for knowledge-management adoption in the SME Sector. Journal of Knowledge Management, v. 9, n. 3, p. 64-82, 2005.

ZIEBA, M.; ZIEBA, K. Knowledge management critical success factors and the innovativeness of KIBS companies. Engineering Economics, v. 25, n. 4, p. 458-465, 2014. 